\title{
Polycomb Silencing Mechanisms in Drosophila
}

\author{
Y.B. Schwartz, T.G. Kahn, G.I. Dellino, and V. Pirrotta \\ Department of Zoology, University of Geneva, CH-1211 Geneva, Switzerland
}

Homeotic genes are a preeminent target for epigenetic mechanisms that program and maintain a chromatin state. The study of their function in Drosophila originally allowed the identification of Polycomb Group (PcG) genes, although we now know that $\mathrm{PcG}$ mechanisms regulate many other genes. Homeotic genes must be expressed in specific segmental domains of the Drosophila body plan throughout development. The expression domains are set in the earliest stages of embryonic development by transient regulators localized in specific regions of the embryo by maternal cues. Shortly after blastoderm, PcG complexes take over to maintain the repressed state in those cells in which the target genes had not been activated in the first 3 hours of development. The descendants of these cells maintain this silent state of the target genes for the rest of development.

PcG proteins are present in all nuclei at all stages in which they have been examined, including the very early embryo, where they are both maternally and zygotically supplied. Remarkably, however, they neither prevent the initial activation of homeotic genes nor their continued expression in the cells in which activation had initially occurred. Both the silenced and the nonsilenced state are therefore epigenetic states that are maintained for the rest of development. After each round of cell division, the gene preserves an epigenetic memory of its chromatin state in the previous cycle. Critical for the establishment and maintenance of this state is the Polycomb Response Element (PRE), the site of action of PcG proteins in the regulatory region of the target genes (Chan et al. 1994). In the case of the Ultrabithorax (Ubx) gene, the best known, the PRE is located 24 kilobases $(\mathrm{kb})$ upstream of the $U b x$ promoter. When the PRE in transgenic construct is excised during development, the repression is lost, showing that it is required not only to establish but to maintain the silenced chromatin state (Busturia et al. 1997).

Despite the attractiveness of this two-state, on-or-off epigenetic model, it is important to bear in mind that the function of PcG complexes does not always adhere to this scheme, which is derived from the regulation of the homeotic genes. A large number of other PcG targets are unlikely to be regulated the same way. Although we know little about most of these other targets, genetic and molecular evidence shows that at least two of them, the engrailed and hedgehog genes, must be reprogrammed at some stages during development (Maurange and Paro 2002). In at least two other cases, the polyhomeotic ( $p h)$ and Posterior sex combs (Psc) genes, themselves encod- ing PcG proteins, the PcG regulation does not define a simple on-or-off alternative. In these genes, the PcG mechanisms down-regulate the expression rather than permanently switching it off (Fauvarque and Dura 1993; Rastelli et al. 1993). How PcG complexes up- and downregulate transcription is even less understood than the allor-none silencing mechanism and most likely depends on the structure of the different PREs and the dynamics of the proteins that interact with them. In this discussion, we will confine ourselves to the behavior of the PREs found in homeotic genes.

\section{THE MAINTENANCE OF THE EPIGENETIC STATE}

An illustration of how the PRE maintains the silenced or nonrepressed state is given in Figure 1, which summarizes the results of reporter constructs containing the $U b x$ promoter fused to lacZ under control of different combinations of early enhancers, imaginal disc enhancers, and PRE (Pirrotta et al. 1995; Poux et al. 1996). The early enhancers of the $U b x$ gene respond to segmentation genes and are activated before the cellular blastoderm stage in the 3-hour-old embryo. They are repressed by the Hunchback segmentation gene product in the anterior half of the embryo. This repression in regions (for simplicity, anterior to parasegment 6) must be maintained since $U b x$ expression in more anterior segments would cause segmental transformations. However, since the Hunchback product disappears shortly after blastoderm, a reporter gene containing only segmental enhancers becomes derepressed and expression occurs in all segments. No expression occurs in the larval imaginal discs since the segmentation genes do not function in larval stages. When an early enhancer is combined with a PRE, permanent repression sets in after blastoderm wherever the reporter gene was inactive at that stage but no repression occurs wherever the gene was active. As a consequence, the segmental domain of expression defined by the early regulatory proteins (posterior to parasegment 6) is maintained.

Imaginal disc enhancers do not function in the early embryo but become activated when the disc primordia are determined. They direct expression in imaginal discs regardless of segmental origin. These enhancers can function therefore in the haltere disc (posterior thorax) but also in the wing (mesothorax) and the eye/antenna (head) discs. If a PRE is added to the imaginal enhancer, there is no expression at any stage because at blastoderm, when 


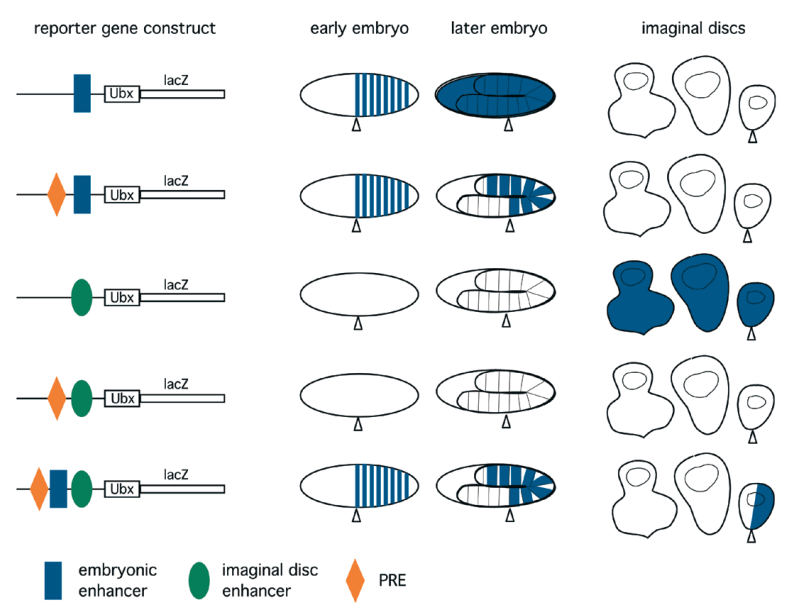

Figure 1. Epigenetic transmission of the repressed state mediated by the PRE. (Left column) Diagrams of reporter constructs containing combinations of early embryonic segmental enhancers, larval imaginal enhancers, and PRE, controlling the expression of the Ubx-lacZ reporter gene. (Right columns) The expression patterns (blue) in the early embryo, later embryo, and in the larval eye antenna, wing, and haltere disk, in anterior-posterior order. (Arrowhead) The parasegment 6 boundary.

silencing is established, the imaginal enhancers are not active. However, if all three elements are present together, the early enhancers activate the gene in the posterior segments, silencing is established only in the anterior segments, and, when the imaginal enhancers become active, they will drive expression only in the segmental domain defined by the early enhancers.

In this way, the PRE could be said to convey the positional information from the early enhancers (which are repressed in the anterior segments) to the larval enhancers (which do not distinguish head and anterior thorax from posterior thorax). There is a gap between the time when the early enhancers cease functioning and when the imaginal enhancers become active. What prevents $\mathrm{PcG}$ silencing from setting in during that time? Current evidence indicates that this is the function of the Trithorax system. Interaction with Trithorax is also mediated by the PRE, which contains a subdomain necessary for Trithorax recruitment (Tillib et al. 1999). While Trithorax also stimulates the expression of the $U b x$ promoter, it appears to maintain a memory of an earlier state of activity that antagonizes the establishment of silencing.

\section{PcG PROTEINS ARE ASSEMBLED IN TWO DISTINCT COMPLEXES}

The effectors of PcG silencing are the multiprotein complexes exemplified by the biochemically purified PRC1 complex, which includes a core quartet of PcG proteins PC, PH, PSC, and dRING (Saurin et al. 2001), and the PRC2 complex, which includes ESC and the histone methyltransferase E(Z) (Cao et al. 2002; Czermin et al. 2002; Kuzmichev et al. 2002 Müller et al. 2002). Both of these complexes are recruited to PREs and both appear to be essential for PcG silencing. Various kinds of genetic, cytogenetic, and biochemical evidence indicate that there is probably considerable variation in the composition of these complexes at different target sites, tissues, or developmental stages.

The targets of the $\mathrm{E}(\mathrm{Z}) / \mathrm{ESC}$ complex are lysine 9 and lysine 27 (K9, K27), which become trimethylated in vitro. Whether both are also methylated in vivo is not clear, but experiments with different antibodies in different laboratories agree that K27 is certainly methylated in vivo at genomic PcG target sites. By analogy with the roles of Suvar3-9 and HP1, it has been proposed that the histone methylation serves to recruit $\mathrm{PC}$ by increasing the affinity of the PC chromodomain for histone H3. In vitro experiments confirm that histone H3 K27 methylation, but not $\mathrm{K} 9$ methylation, increases the in vitro binding of the PC chromodomain to a peptide containing the K27 region about 100-fold (Fischle et al. 2003). The increase in binding to full-length recombinant $\mathrm{H} 3$ me3K27 is less dramatic (Czermin et al. 2002), probably because PC has additional affinity for intact $\mathrm{H} 3$ not mediated by the chromodomain (Breiling et al. 1999).

One current model envisions the early recruitment of the $E(Z)$ complex in the early embryo as the first step in the establishment of $\mathrm{PcG}$ silencing. This recruitment would depend on DNA-binding proteins that recognize specific sequences in the PRE. The $\mathrm{E}(\mathrm{Z})$-dependent histone $\mathrm{H} 3$ methylation at $\mathrm{K} 27$ would then be necessary and sufficient for the subsequent recruitment of the PC-containing complex by the affinity of $\mathrm{PC}$ for the methylated H3. Alternatively, DNA-binding recruiters have been suggested to act cooperatively to recruit various components of the $\mathrm{E}(\mathrm{Z})$ complex and of the PC complex.

\section{EVIDENCE FOR RECRUITMENT}

PREs are DNA sequences spread over 500-1000 base pairs (bp) within which a functional minimum core region of a few hundred base pairs is capable of recapitulating most of the silencing activities when included in a reporter 
construct (Chan et al. 1994; Horard et al. 2000). The additional flanking sequences appear to contribute to the stability of the silenced state. Transposon constructs containing PRE core sequences bind PcG proteins that can be visualized at the site of insertion in polytene chromosomes and can be detected by chromatin immunoprecipitation. These core sequences contain certain recurring motifs, some of which are known to be consensus sequences for DNA-binding proteins, but there is otherwise little sequence homology between different PREs. Two such motifs are the GAGAG consensus binding sites for GAGA factor and the GCCAT consensus for Pleiohomeotic (PHO), the fly homolog of the mammalian YY1 factor (Brown et al. 1998; Horard et al. 2000). A third motif frequently associated with PREs is the YGAGYG consensus that binds Zeste (Benson and Pirrotta 1987, 1988). However, Zeste function is not required for PcG silencing and Zeste binding sites can be separated from PREs without impairing their silencing activity. On the contrary, the addition of Zeste binding sites generally stimulates the expression of PRE-containing reporter constructs in a Zestedependent manner (Horard et al. 2000), consistent with the fact that Zeste has been shown to recruit the Brahma chromatin-remodeling complex (Kal et al. 2000). Because of their prominent presence in multiple copies in most PRE sequences, the PHO and GAGA binding sites have been proposed to bind proteins that contribute to the recruitment of PcG components that have themselves no specific DNA-binding activity.

\section{GAGA Factor}

GAGAG motifs contained in PREs have been found to bind GAGA factor but also Pipsqueak, both BTB domain proteins important for PcG silencing (Hagstrom et al. 1997; Busturia et al. 2001; Hodgson et al. 2001; Americo et al. 2002; Huang et al. 2002). Both of these proteins have been found to be localized at homeotic loci on polytene chromosomes, and mutations in either enhance homeotic phenotypes of PcG mutations, implying that they contribute to PcG silencing. However, GAGAG motifs are found at many promoters, including those of homeotic genes but also those of many other genes such as heat shock genes, which are not known to be repressed by PcG mechanisms.

A PcG complex biochemically purified from Drosophila embryos was not found to contain GAGA factor or Pipsqueak although it apparently included a number of other proteins in addition to the core PcG components. However, coimmunoprecipitation experiments with embryonic nuclear extracts have shown that GAGA factor isoforms can associate with protein complexes containing PC. Furthermore, PC-containing complexes present in the nuclear extracts can bind in vitro to DNA fragments from the bxd PRE and the binding is specifically competed by oligonucleotides containing GAGA factor binding sites (Horard et al. 2000). We conclude that a variety of PcG complexes exist in vivo, a subset of which does contain GAGA factor which could act as a recruiter of PcG components to the PRE. It is not sufficient, however. GAGA factor binding sites are present at a very large number of genomic sites in contexts that bear no relationship to PcG silencing. We suppose therefore that GAGA factor is one of several recruiters whose concerted action brings together and eventually stabilizes a PcG complex to the PRE.

\section{PHO Factor}

PHO and its related protein PHO-like are necessary for PcG silencing (Fritsch et al. 1999; Brown et al. 2003). Coimmunoprecipitation experiments have detected PHO in association with $\mathrm{E}(\mathrm{Z}) / \mathrm{ESC}$ complexes but not with $\mathrm{PC}$ complexes in most embryonic nuclear extracts (Poux et al. 2001a; but see also Poux et al. 2001b). However, PHO is not found in the best characterized form of the $\mathrm{E}(\mathrm{Z}) / \mathrm{ESC}$ complex, the $600-\mathrm{kD}$ complex. Here too, however, other and higher molecular weight complexes have been identified and it is possible that they include PHO. Other studies have also detected physical interactions between PHO and PC (Mohd-Sarip et al. 2002). These discrepant reports raise again the possibility that different types of complexes and different interactions may exist in different cells at different developmental stages or may be detected under different in vitro conditions.

\section{MULTIPLE DNA-BINDING RECRUITERS}

The analysis of PRE sequences indicates that different DNA-binding proteins play a role in their function. The fact that two proteins bind to each of the two best characterized motifs could explain why mutations in the GAGA factor, Pipsqueak, PHO, and PHO-like have relatively mild effects on PcG silencing, if each can be at least partly replaced by its related DNA-binding protein. Other sequence motifs are likely to be important since synthetic combinations of GAGA and PHO binding sites are not sufficient to act as PREs. Different PREs share no extended sequence homology but Ringrose et al. (2003), using a whole genome approach, found that an algorithm that searches for clusters of the GAGA, PHO, and Zeste binding motifs identified most known PREs and predicted successfully many others. An analysis of these sequences showed that a few other motifs are frequently associated with these sites, suggesting that additional DNA-binding proteins might be implicated. It is possible therefore that the loss of one of several DNA-binding recruiters of $\mathrm{PcG}$ proteins might have only weak effects in the overall recruitment process.

\section{CONSERVATION OF PRE SEQUENCES ACROSS SPECIES}

Another approach often used to identify functionally important sequence elements is a phylogenetic comparison. The sequences of the bxd PREs from four Drosophila species-D. melanogaster, D. eugracilis, D. virilis (Dellino et al. 2002), and D. pseudoobscurashow that conservation is extensive and remarkable over an interval of almost one kilobase, in view of the fact that $D$. virilis and $D$. pseudoobscura are thought to have diverged from $D$. melanogaster between 40 and 60 million years ago. Figure 2 compares the core PRE region from 


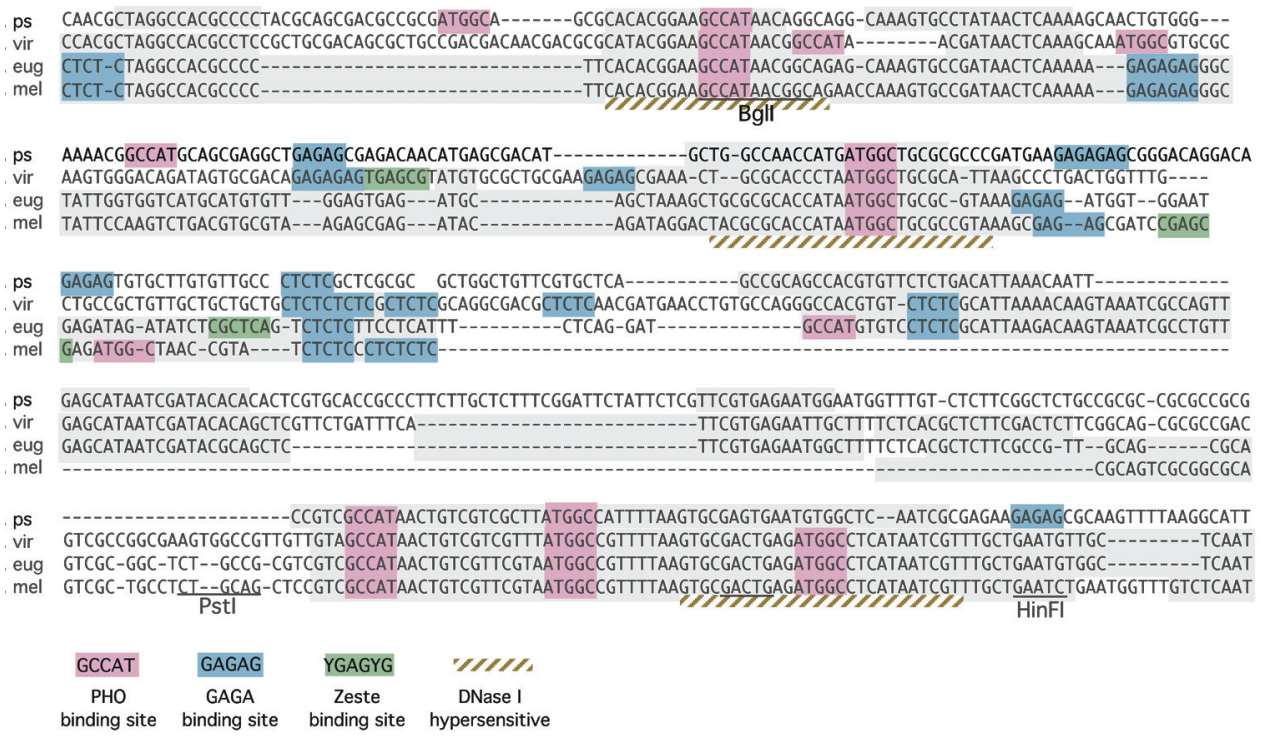

Figure 2. Conservation of bxd PRE sequences among Drosophila species. The sequences of the core PRE region from $D$. melanogaster, $D$. eugracilis, $D$. virilis, and $D$. pseudoobscura are aligned for maximum homology. (Dotted lines) Sequences that have been inserted or deleted in different species. Consensus sequences for known DNA-binding proteins are shown in blue (GAGA), red (PHO), and green (Zeste). (Gray blocks) Highly conserved blocks; (brown barred lines) the DNase I hypersensitive regions. For reference, three restriction sites present in the D. melanogaster sequence are indicated: BglI, PstI, and HinFI. The D. pseudoobscura sequence was obtained from the Baylor Drosophila Genome Project (http://www.hgsc.bcm.tmc.edu/ projects/drosophila/). The three other sequences are from Dellino et al. (2002).

the four species, showing that the GAGA and PHO binding sites are either strictly conserved or where one is lost through mutation, it is recreated nearby by compensatory mutations. Even more remarkable is the complete conservation of some sequence intervals over more than $100 \mathrm{nt}$ although they do not contain any identified DNA-binding motifs. The sequence conservation is reflected by the conservation of a set of four prominent DNase I hypersensitive sites within a conserved sequence context. These results support the idea that PREs are targets for a number of DNA-binding proteins, of which we know at present only a few, but which act together to recruit PcG components. However, the extensive sequence conservation makes it difficult to identify potential DNA-binding motifs other than those already known.

The four DNase I hypersensitive sites are clustered within $<400 \mathrm{bp}$ in the PRE core, making it unlikely that the PRE core region could be assembled in nucleosomes. The presence of ten GAGA factor binding sites in this interval is also incompatible with nucleosome formation, in view of the cooperative binding properties of GAGA factor (Katsani et al. 1999) and its known propensity to reposition nucleosomes (Tsukiyama et al. 1994). The PRE core region is also highly accessible to restriction enzymes (Dellino et al. 2004).

To obtain more detailed information on the chromatin structure of the PRE core region, we carried out genomic footprinting using ligation-mediated PCR. DNase I footprinting was done in parallel with chromatin from wildtype embryos or from esc-lesc- embryos produced by esc-lesc- mothers, hence lacking both maternal and zygotic ESC and unable to establish PcG silencing. Figure 3 shows that the hypersensitive sites are preserved in the mutant embryos but a pattern of relatively more protected, footprint-like regions becomes more DNase I sensitive in the mutant. A similar change, though less pronounced, was seen with chromatin from embryos carrying the homozygous $E(z)^{\mathrm{S} 2}$ temperature-sensitive mutation, raised at nonpermissive temperature. The fact that neither the esc nor the $E(z)$ mutation affect the DNase I hypersensitive sites, suggests that they are due to the DNA-binding proteins such as GAGA factor or PHO. The changes induced by the mutations probably indicate sequences protected by the recruited $\mathrm{PcG}$ proteins.

\section{TARGETING OF PcG COMPLEXES BY DNA- BINDING DOMAIN FUSIONS}

Dissection of the recruitment process has been attempted using fusions of different PcG proteins with exogenous DNA-binding domains to target the proteins to a reporter gene containing the corresponding DNA-binding sites (Müller 1995; Poux et al. 2001a,b). The rationale of these experiments was to bypass part of the recruitment process and ask which protein, when targeted to a $U b x-$ lac $Z$ reporter gene, can recruit the remaining components necessary to establish functional silencing. These experiments showed that PC, PH, PSC, or ESC can all silence the reporter gene to which they are targeted in a manner dependent on the other PcG components, implying that 


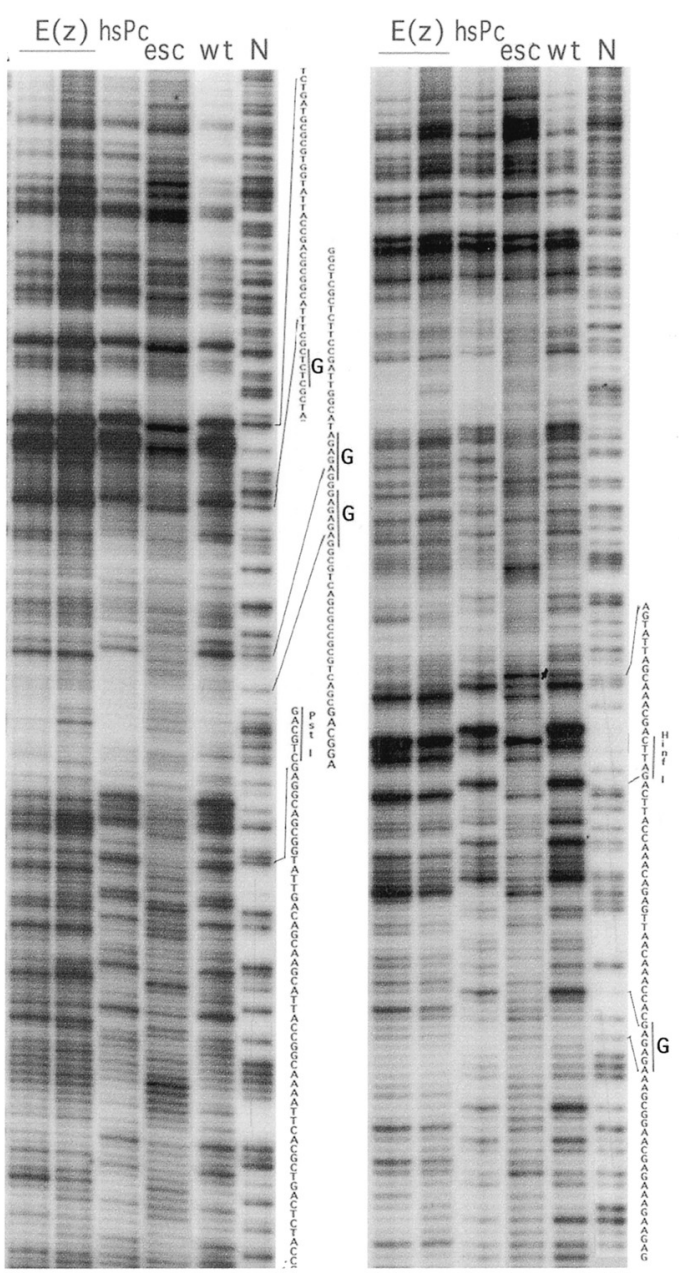

Figure 3. Genomic footprinting of the $b x d$ PRE core region. Embryonic chromatin was partially digested with DnaseI and the DNA was then amplified by ligation-mediated PCR. The product was analyzed by short (left) or long (right) runs of gel electrophoresis. The lanes show the wild-type (wt), $E(z)^{\mathrm{S} 2}$ temperature-sensitive mutants treated at nonpermissive temperature $4 \mathrm{hr}$ or overnight $(\mathrm{E}(\mathrm{z}))$, esc embryos from esc mothers (esc), or embryos overexpressing $\mathrm{PC}$ protein $(\mathrm{hsPc})$. $\mathrm{N}$ indicates free DNA. The sequence is approximately aligned with the bands. PstI and HinF1 restriction sites and GAGA consensus sequences $(\mathrm{G})$ are indicated. Note that the $E(z)$ and esc sequences contain an insertion of two nucleotides relative to the others.

they can recruit the other necessary proteins. These experiments revealed two surprising facts. One is that, although the reporter gene was correctly repressed in the embryo, the silenced state was not maintained during larval development. The second was that targeted ESC and targeted PC establish silencing dependent on the endogenous $P c$ or $e s c$ gene, respectively, implying that each can recruit the other although they appear to belong to different, noncoimmunoprecipitating complexes.

These results imply that there must be an early stage at which PC-containing complexes interact with ESC-containing complexes. When we prepared nuclear extracts from 0-3-hour-old embryos, we found, in fact, that PC coimmunoprecipitates with ESC, E(Z), and PHO (Poux et al. 2001b). In contrast, at later embryonic stages, PC no longer appears to interact directly with the $\mathrm{ESC} / \mathrm{E}(\mathrm{Z}) \mathrm{com}$ plex. If both complexes must be present for silencing to occur, these results would explain why tethered PC or tethered ESC can silence a reporter gene at earlier stages but not during later development. They also imply that the presence of the $\mathrm{ESC} / \mathrm{E}(\mathrm{Z})$ histone methyltransferase complex is not sufficient to recruit $\mathrm{PC}$ complexes at later stages.

The $\operatorname{ESC} / \mathrm{E}(\mathrm{Z})$ complex is essential for the establishment silencing. Genetic experiments show that esc function is needed only in the early embryo, within the first 4 hours of development (Struhl and Brower 1982; Simon et al. 1995), while during later embryonic development esc transcription is said to disappear. A sufficient maternal endowment of ESC protein can support the development of homozygous esc embryos and lead to nearly normal adults. Since ESC is a constituent of the E(Z) histone H3 methyltransferase complex and is required for its enzymatic activity, how is methylation maintained in the absence of ESC in later development? Current work indicates that although esc expression ceases during embryonic development, a second esc gene, esc-like, takes over to ensure continued H3 K27 methylation (D. McCabe et al., unpubl.).

\section{DISTRIBUTION OF PcG PROTEINS AND HISTONE H3 METHYLATION IN THE REPRESSED $U b x$ GENE}

To test the role of histone methylation in the recruitment and localization of $\mathrm{PcG}$ proteins at a repressed gene, we determined the distribution of $\mathrm{H} 3$ me3K27 and of $\mathrm{PcG}$ proteins on various target genes or transgene constructs in embryos and in tissue culture cells (Y.B. Schwartz et al., in prep.). In brief, these chromatin immunoprecipitation experiments showed that $\mathrm{PC}, \mathrm{PSC}$, and $\mathrm{E}(\mathrm{Z})$ proteins are primarily localized over the PRE of the silenced $U b x$ gene, although a weak but significant association of $\mathrm{PC}$ can be detected over much of the gene. In contrast, H3 me3K27 is broadly distributed over the entire $75 \mathrm{~kb}$ of the $U b x$ transcription unit and at least $40 \mathrm{~kb}$ of upstream regulatory region. Only the PRE core itself appears to be unmethylated and, in fact, depleted of histone $\mathrm{H} 3$, in agreement with the idea that it is probably nucleosome-free. These observations support the idea that DNA-binding proteins are the primary recruiters of PcG complexes at the PRE and that H3 methylation plays an important but subsequent role. They raise the question of what this role might be and how methylation takes place over such an extended domain if the $\mathrm{E}(\mathrm{Z})$ methyltransferase is localized at the PRE.

$\mathrm{H} 3$ methylation is important for the stability and repressive function of the PcG complex. Loss of the $\mathrm{E}(\mathrm{Z})$ methyltransferase function during development eventually causes the derepression of homeotic genes and the dissociation of $\mathrm{PcG}$ proteins from most of their binding sites on polytene chromosomes (Rastelli et al. 1993; Beuchle et al. 2001). It is not simply needed for binding of the PC complex since a requirement for ESC and E(Z) is still observed even when PC is directly recruited to a reporter gene by fusion with a DNA-binding domain.

The fact that the PRE is essential for maintenance of silencing implies that the repressed state is not just perpet- 
uated by modification of a silenced chromatin region or by the persistence of a protein complex that acts as a template. The broad distribution of the H3 K27 methylation over the silenced gene also implies that methylation is not sufficient for the stable recruitment of $\mathrm{PcG}$ complexes. Histone methylation might have several possible roles. It might be necessary to stabilize the association of the PcG complex with the PRE. It might allow the spreading of PcG complex assembly, extending from the PRE to cover a larger chromatin domain. It might induce a switch in the PcG complex that permits it to repress promoter function. It might simply act as the carrier of the epigenetic memory by facilitating the reassembly of the complex at sites that were methylated in the previous cell cycle. Chromatin immunoprecipitation results do not support a uniform, continuous spreading of the PcG complex fed in from an entry site (the PRE) and coating the chromatin over an extensive domain, such as is thought to occur in the case of the yeast SIR complex (Hecht et al. 1996). However, they would be consistent with a more transient interaction of PcG complex mediated by histone methylation according to two scenarios (Fig. 4). One is a looping mechanism by which the complex bound at the PRE loops over to interact transiently with nucleosomes. This interaction would be facilitated by the affinity of PC for the methylated nucleosomes without stable binding to any one sequence for any length of time. At the same

A

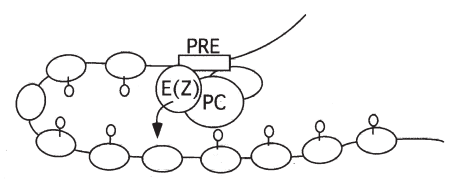

B

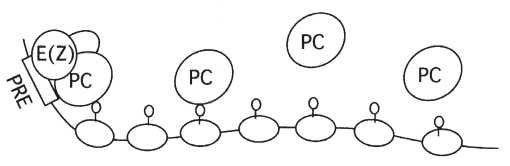

C

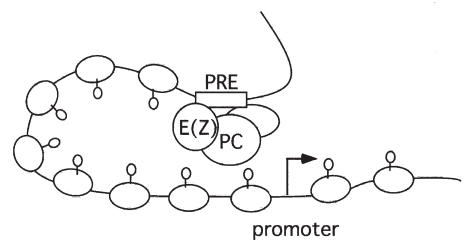

Figure 4. Three possible roles of histone methylation. $(A)$ The chromodomain of PC bound at the PRE mediates transient interactions with methylated nucleosomes by a looping mechanism that allows the PRE complex to scan a large region, maintaining the fully methylated state. $(B)$ The affinity of PC for methylated nucleosomes maintains a local high concentration of $\mathrm{PC}$ protein, stabilizing the PRE complex. A similar mechanism helps to rebind to between previously repressed genes every cell cycle. $(C)$ The transient looping mediated by PC interaction with methylated nucleosomes also brings the PRE complex in the vicinity of the promoter, allowing it to block the promoter complex, if present, or prevent its recruitment by blocking nucleosome remodeling. The ball and stick indicates a methylated H3 lysine 27. time, it would allow a large domain to be constantly surveyed by the $E(Z)$ complex bound at the PRE, thus maintaining the fully methylated state. In another view, the interaction of PC with the methylated histone would contribute to retain a local concentration of $\mathrm{PC}$ in the vicinity of the PRE. The binding of chromatin proteins such as HP1, like that of DNA-binding proteins, has been shown by photobleaching techniques to be short-lived with residence times of the order of seconds (Cheutin et al. 2003; Phair et al. 2004). The transient binding of PC to a methylated chromatin domain could maintain a local high concentration of $\mathrm{PC}$ protein to ensure rebinding to the PRE. Both mechanisms might, in fact, help to stabilize the presence of the PcG complex and to mediate the widespread methylation. The large methylated domain in turn contributes to the stability of the PcG complex mediated by the interaction of the PC chromodomain with the methylated histone and ensures the persistence of the epigenetic mark from one round of DNA replication to the next if the old nucleosomes are randomly distributed between the two daughter DNA molecules.

\section{SILENCING MECHANISMS}

The traditional view of chromatin silencing presupposed that the affected chromatin would be highly condensed. In this view, silencing would result from the inability of transactivators and transcriptional machinery to have access to the condensed chromatin. This type of mechanism implies that condensation is responsible for silencing rather than the reverse. To test this idea, we have recently used a reporter construct in which the bxd PRE was placed next to the hsp26 promoter (Dellino et al. 2004). As a result, the $h s p 26$ promoter becomes repressed and unable to be induced in a high proportion of the cells of animals carrying the transgene.

The heat shock promoter is normally preset by the cooperative binding of GAGA factor, TBP, and RNA Pol II, which together position a nucleosome and leave DNase Isensitive sites over the heat shock elements. The polymerase is known to initiate transcription but to be unable to elongate beyond 25-50 nt in the absence of HSF. At the repressed $h s p 26$ promoter, the nucleosome is still correctly positioned and chromatin immunoprecipitation showed that both TBP and RNA polymerase were able to bind to the promoter and, upon heat shock, the HSF had normal access to the heat shock elements. The analysis of the promoter by permanganate sensitivity showed that, although the polymerase is bound to the promoter even without heat shock, it fails to open the strands and initiate transcription. Therefore, these results imply that, at least at this promoter, PcG silencing does not prevent access of the promoter factors but acts directly on the promoter complex to prevent initiation. This does not exclude the possibility that in other promoter contexts the PcG complex might also inhibit certain kinds of chromatin remodeling necessary to make the promoter accessible to the transcription machinery, as has been proposed by Shao et al. (1999). A similar direct effect on the promoter complex has been found in yeast, where the SIR-silencing complexes do not prevent the binding of 
promoter factors and RNA polymerase but inhibit productive transcription (Sekinger and Gross 2001)

To account for our observations we envision two possible scenarios. Both depend on the looping mechanism described above. One scenario would view the methylated nucleosomes over the chromatin domain as providing a ready access for the PcG complex to interact with the promoter region. This interaction would be transient unless the PcG complex meets with promoter components for which it has specific affinities. Evidence for such affinities is the finding of several TAFs copurifying with the PRC1 complex as well as the observation that PcG components coimmunoprecipitate with TBP (Breiling et al. 2001; Saurin et al. 2001). This would be the situation at promoters that have independent mechanisms for recruiting general transcription factors, such as heat shock promoters, where the chromatin configuration is permanently preset by the cooperative binding of GAGA factor, TBP, and RNA polymerase. However, different promoters recruit promoter factors in different ways. In the case of the $U b x$ promoter, in particular, we know nothing about this mechanism except that, as a TATAless promoter, it is presumed to require help from activators and other mechanisms. Inhibition of chromatin remodeling might also play a role at such sites.

\section{CONCLUSIONS}

The study of PcG silencing mechanisms has made enormous progress over the last 10 years and has revealed an extraordinary degree of complexity in the molecular mechanisms that recruit the complexes, establish silencing, and maintain the epigenetic silent state. More complexity is certain to be revealed in the future. The phylogenetically most ancient elements of the mechanism are the $\mathrm{E}(\mathrm{Z}) / \mathrm{ESC}$ components that appear to antedate all others and function in epigenetic silencing in plants (Goodrich et al. 1997; Köhler et al. 2003). In animals, the PC and associated components were added to work in concert with the histone methyltransferase complex and their complex interactions have been conserved at least from insects to mammals.

We might wonder why this large array of components and complex recruiting mechanisms is necessary. If it is sufficient to provide a PcG protein with a DNA-binding domain in order to assemble the functional silencing complex, why has nature not provided this simple solution? One explanation might be that the assembly of the repressive complexes must be carefully regulated. The PcG proteins are present at all times but they must not prevent the activation of the target genes in their appropriate domains. The assembly must be designed to be exquisitely sensitive to the chromatin state of the target gene so that an active gene does not become silenced. Other explanations might be that different components of the silencing mechanism may have special roles, not necessarily in association with the others, but participate in the $\mathrm{PcG}$ complexes to provide stability, flexibility, and additional regulatory features. The role of PcG proteins in the regulation of many other genes in addition to their classical targets, the homeotic genes, will be the object of research for the future.

\section{ACKNOWLEDGMENTS}

This work was supported by grants from the Human Frontiers Science Program, from the Swiss National Science Foundation, and by the "Frontiers in Genetics" Pôle de Recherche National of the Swiss National Science Foundation.

\section{REFERENCES}

Americo G., Whiteley M., Brown J.L., Fujioka M., Jaynes J.B., amd Kassis J.A. 2002. A complex array of DNA-binding complexes required for pairing-sensitive silencing by a polycomb group response element from the Drosophila engrailed gene. Genetics 160: 1561.

Benson M. and Pirrotta V. 1987. The product of the Drosophila zeste gene binds to specific DNA sequences in white and $U b x$. EMBO J. 6: 1387.

. 1988. The Drosophila zeste protein binds cooperatively to sites in many gene regulatory regions: Implications for transvection and gene regulation. EMBO J. 7: 3907.

Beuchle D., Struhl G., and Müller J. 2001. Polycomb group proteins and heritable silencing of Drosophila Hox genes. Development 128: 993.

Breiling A., Turner B.M., Bianchi M.E., and Orlando V. 2001. General transcription factors bind promoters repressed by Polycomb group proteins. Nature 412: 651.

Breiling A.E., Bonte E., Ferrari S., Becker P.B., and Paro R. 1999. The Drosophila polycomb protein interacts with nucleosomal core particles in vitro via its repression domain. Mol. Cell. Biol. 19: 8451

Brown J.L., Fritsch C., Müller J., and Kassis J.A. 2003. The Drosophila pho-like gene encodes a YY1-related DNA binding protein that is redundant with pleiohomeotic in homeotic gene silencing. Development 130: 285.

Brown J.L., Mucci D., Whiteley M., Dirksen M.L., and Kassis J.A. 1998. The Drosophila Polycomb group gene pleiohomeotic encodes a DNA binding protein with homology to the transcription factor YY1. Mol. Cell 4: 1057.

Busturia A., Wightman C.D., and Sakonju S. 1997. A silencer is required for maintenance of transcriptional repression throughout Drosophila development. Development 124: 4343.

Busturia A., Lloyd A., Bejarano F., Zavortink M. Xin H., and Sakonju S. 2001. The MCP silencer of the Drosophila Abd-B gene requires both Pleiohomeotic and GAGA factor for the maintenance of repression. Development 128: 2163.

Cao R., Wang L., Wang H., Xin L., Erdjument-Bromage H., Tempst P., Jones R.S., and Zhang Y. 2002. Role of histone H3 lysine 27 methylation in Polycomb-group silencing. Science 298: 1039.

Chan C.-S., Rastelli L., and Pirrotta V. 1994. A Polycomb response element in the $U b x$ gene that determines an epigenetically inherited state of repression. EMBO J. 13: 2553.

Cheutin T., McNairn A., Jenuwein T., Gilbert D.M., Singh P.B., and Misteli T. 2003. Maintenance of stable heterochromatin domains by dynamic HP1 binding. Science 299: 721.

Czermin B., Melfi R., McCabe D., Seitz V., Imhof A., and Pirrotta V. 2002. Drosophila enhancer of Zeste/ESC complexes have a histone $\mathrm{H} 3$ methyltransferase activity that marks chromosomal Polycomb sites. Cell 111: 185.

Dellino G.I., Tatout C., and Pirrotta V. 2002. Conservation of sequences and chromatin structure of the bxd polycomb response element among Drosophila species. Int. J. Dev. Biol. 46: 133.

Dellino G.I., Schwartz Y.B., Farkas G., McCabe D., Elgin S.C.R., and Pirrotta V. 2004. Polycomb silencing blocks transcription initiation. Mol. Cell 13: 887.

Fauvarque M.-O. and Dura J.-M. 1993. polyhomeotic regulatory sequences induce developmental regulator-dependent variegation and targeted P-element insertions in Drosophila. Genes Dev. 7: 1508. 
Fischle W., Wang Y., Jacobs S.A., Kim Y., Allis C.D., and Khorasanizadeh S. 2003. Molecular basis for the discrimination of repressive methyl-lysine marks in histone $\mathrm{H} 3$ by Polycomb and HP1 chromodomains. Genes Dev. 17: 1870.

Fritsch C., Brown J.L., Kassis J.A., and Müller J. 1999. The DNA-binding polycomb group protein pleiohomeotic mediates silencing of a Drosophila homeotic gene. Development 126: 3905 .

Goodrich J., Puangsomlee P., Martin M., Long D., Meyerowitz E., and Coupland G. 1997. A Polycomb-group gene regulates homeotic gene expression in Arabidopsis. Nature 386: 44.

Hagstrom K., Müller M., and Schedl P. 1997. A Polycomb and GAGA dependent silencer adjoins the Fab7 boundary in the Drosophila bithorax complex. Genetics 146: 1365.

Hecht A., Strahl-Bolsinger S., and Grunstein M. 1996. Spreading of transcriptional repressor SIR3 from telomeric heterochromatin. Nature 383: 92.

Hodgson J.W., Argiropoulos B., and Brock H.W. 2001. Sitespecific recognition of a 70-base-pair element containing $\mathrm{d}(\mathrm{GA})(\mathrm{n})$ repeats mediates bithoraxoid polycomb group response element-specific silencing. Mol. Cell. Biol. 21: 4528.

Horard D., Tatout C., Poux S., and Pirrotta V. 2000. Structure of a polycomb response element and in vitro binding of polycomb group complexes containing GAGA factor. Mol. Cell. Biol. 20: 3187.

Huang D.-H., Chang Y.-L., Yang C.-C., Pan I.-C., and King B. 2002. pipsqueak encodes a factor essential for sequence-specific targeting of a polycomb group protein complex. Mol. Cell. Biol. 22: 6261 .

Kal A.J., Mahmoudi T., Zak N.B., and Verrijzer C.P. 2000. The Drosophila brahma complex is an essential coactivator for the trithorax group protein zeste. Genes Dev. 14: 1058.

Katsani K.R., Hajibagheri N., and Verrijzer C.P. 1999. Co-operative DNA binding by GAGA transcription factor requires the conserved BTB/POZ domain and reorganizes promoter topology. EMBO J. 18: 698.

Kuzmichev A., Nishioka K., Erdjument-Bromage H., Tempst P., and Reinberg D. 2002. Histone methyltransferase activity associated with a human multiprotein complex containing the Enhancer of Zeste protein. Genes Dev. 22: 2893.

Köhler C., Hennig L., Spillane C., Pien S., Gruissem W., and Grossniklaus U. 2003. The Polycomb-group protein MEDEA regulates seed development by controlling expression of the MADS-box gene PHERES1. Genes Dev. 17: 1540.

Maurange C. and Paro R. 2002. A cellular memory module conveys epigenetic inheritance of hedgehog expression during Drosophila wing imaginal disc development. Genes Dev. 16: 2672.

Mohd-Sarip A.F., Venturini F., Chalkley G.E., and Verrijzer C.P. 2002. Pleiohomeotic can link polycomb to DNA and mediate transcriptional repression. Mol. Cell. Biol. 22: 7473.

Müller J. 1995. Transcriptional silencing by the Polycomb protein in Drosophila embryos. EMBO J. 14: 1209.
Müller J., Hart C.M., Francis N.J., Vargas M.L., Sengupta A., Wild B., Miller E.L., O'Connor M.B., Kingston R.E., and Simon J.A. 2002. Histone methyltransferase activity of a Drosophila Polycomb group repressor complex. Cell 111: 197.

Phair R.D., Scaffidi P., Elbi C., Vecerova J., Dey A., Ozato K., Brown D.T., Hager G., Bustin M., and Misteli T. 2004. Global nature of dynamic protein-chromatin interactions in vivo: Three-dimensional genome scanning and dynamic interaction networks of chromatin proteins. Mol. Cell. Biol. 24: 6393.

Pirrotta V., Chan C.-S., McCabe D., and Qian S. 1995. Upstream parasegmental enhancers and the establishment of the expression pattern of the Ubx gene. Genetics 141: 1439.

Poux S., Kostic C., and Pirrotta V. 1996. Hunchback-independent silencing of late $U b x$ enhancers by a Polycomb Group Response Element. EMBO J. 15: 4713.

Poux S., McCabe D., and Pirrotta V. 2001a. Recruitment of components of Polycomb Group chromatin complexes in Drosophila. Development 128: 75.

Poux S., Melfi R., and Pirrotta V. 2001b. Establishment of Polycomb silencing requires a transient interaction between PC and ESC. Genes Dev. 15: 2509.

Rastelli L., Chan C.S., and Pirrotta V. 1993. Related chromosome binding sites for zeste, suppressors of zeste and Polycomb group proteins in Drosophila and their dependence on Enhancer of zeste function. EMBO J. 12: 1513.

Ringrose L., Rehmsmeier M., Dura J.M., and Paro R. 2003. Genome-wide prediction of Polycomb/Trithorax response elements in Drosophila melanogaster. Dev. Cell 5: 759.

Saurin A.J., Shao Z., Erdjument-Bromage H., Tempst P., and Kingston R.E. 2001. A Drosophila Polycomb group complex includes Zeste and dTAFII proteins. Nature 412: 655.

Sekinger E.A. and Gross D.S. 2001. Silenced chromatin is permissive to activator binding and PIC recruitment. Cell 105: 403.

Shao Z., Raible F., Mollaaghababa R., Guyon J.R., Wu C.T., Bender W., and Kingston R.E. 1999. Stabilization of chromatin structure by PRC1, a Polycomb complex. Cell 98: 37.

Simon J., Bornemann D., Lunde K., and Schwartz C. 1995. The extra sex combs product contains WD40 repeats and its time of action implies a role distinct from other Polycomb group products. Mech. Dev. 53: 197.

Struhl G. and Brower D. 1982. Early role of the esc+ gene product in the determination of segments in Drosophila. Cell 31: 285.

Tsukiyama T., Becker P.B., and Wu C. 1994. ATP-dependent nucleosome disruption at a heat-shock promoter mediated by binding of GAGA transcription factor. Nature 367: 525.

Tillib S., Petruk S., Sedkov Y., Kuzin A., Fujioka M., Goto T., and Mazo A. 1999. Trithorax- and Polycomb-group response elements within an Ultrabithorax transcription maintenance unit consist of closely situated but separable sequences. Mol. Cell. Biol. 19: 5189. 


\section{$8_{\mathrm{CSH}}^{\infty} \mathrm{H} \&$ Cold Spring Harbor Symposia SYMPOSIA On Quantitative Biology}

\section{Polycomb Silencing Mechanisms in Drosophila}

Y.B. SCHWARTZ, T.G. KAHN, G.I. DELLINO, et al.

Cold Spring Harb Symp Quant Biol 2004 69: 301-308

Access the most recent version at doi:10.1101/sqb.2004.69.301

References This article cites 47 articles, 25 of which can be accessed free at: http://symposium.cshlp.org/content/69/301.full.html\#ref-list-1

\section{License}

Email Alerting Receive free email alerts when new articles cite this article - sign up in Service the box at the top right corner of the article or click here. 